\title{
Clinical applications of custom-made vaginal cylinders constructed using three-dimensional printing technology
}

\author{
Rajni Sethi, MD, Adam Cunha, PhD, Katherine Mellis, BS, Timmy Siauw, PhD, Chris Diederich, PhD, Jean Pouliot, PhD, \\ I-Chow Hsu, MD \\ Department of Radiation Oncology. University of California San Francisco, San Francisco, CA, USA
}

\begin{abstract}
Purpose: Three-dimensional (3D) printing technology allows physicians to rapidly create customized devices for patients. We report our initial clinical experience using this technology to create custom applicators for vaginal brachytherapy.

Material and methods: Three brachytherapy patients with unique clinical needs were identified as likely to benefit from a customized vaginal applicator. Patient 1 underwent intracavitary vaginal cuff brachytherapy after hysterectomy and chemotherapy for stage IA papillary serous endometrial cancer using a custom printed $2.75 \mathrm{~cm}$ diameter segmented vaginal cylinder with a central channel. Patient 2 underwent interstitial brachytherapy for a vaginal cuff recurrence of endometrial cancer after prior hysterectomy, whole pelvis radiotherapy, and brachytherapy boost. We printed a $2 \mathrm{~cm}$ diameter vaginal cylinder with one central and six peripheral catheter channels to fit a narrow vaginal canal. Patient 3 underwent interstitial brachytherapy boost for stage IIIA vulvar cancer with vaginal extension. For more secure applicator fit within a wide vaginal canal, we printed a $3.5 \mathrm{~cm}$ diameter solid cylinder with one central tandem channel and ten peripheral catheter channels. The applicators were printed in a biocompatible, sterilizable thermoplastic.

Results: Patient 1 received 31.5 Gy to the surface in three fractions over two weeks. Patient 2 received 36 Gy to the CTV in six fractions over two implants one week apart, with interstitial hyperthermia once per implant. Patient 3 received 18 Gy in three fractions over one implant after 45 Gy external beam radiotherapy. Brachytherapy was tolerated well with no grade 3 or higher toxicity and no local recurrences.

Conclusions: We established a workflow to rapidly manufacture and implement customized vaginal applicators that can be sterilized and are made of biocompatible material, resulting in high-quality brachytherapy for patients whose anatomy is not ideally suited for standard, commercially available applicators.
\end{abstract}

Key words: 3D printing, brachytherapy, endometrial, vaginal cuff, vulvar.

\section{Purpose}

In an effort to improve applicator fit and resulting implant geometry, we used three-dimensional (3D) printing technology as a way to rapidly manufacture customized brachytherapy applicators for women undergoing vaginal brachytherapy. Poor applicator fit during vaginal intracavitary brachytherapy can lead to air gaps and under-dosing of the target volume [1,2]. For interstitial implants, poor applicator fit may lead to poor implant geometry and increased inter-fraction variability when multiple fractions are delivered over a single implant [3]. While commercial applicators are available in a variety of sizes, we desired further flexibility in applicator shape and structure to improve fit within a cavity or allow optimal needle positioning for interstitial technique. While other centers have documented interest in personalized vaginal applicators $[4,5]$ and one group has reported using stereolithography to create vaginal applicators [6], we know of no prior experience using rapid prototyping with $3 \mathrm{D}$ in conjunction with an interstitial technique.

Medical applications of 3D printing in development include medical modeling for maxillofacial surgical management $[7,8]$ bone reconstructions $[9,10]$, and oral surgeries [11]. The precision of 3D printers has been closely evaluated for medical applications with several studies confirming high levels of precision [12,13,14]. Manufacturers have supported medical interests in $3 \mathrm{D}$ printing by 
introducing printing materials that pass the International Standard ISO-10993 as well as the United States Pharmacopeia (USP) standards for biocompatibility.

In this article, we present the clinical implementation of rapid prototype 3D printed custom, biocompatible, sterilizable vaginal applicators at our institution, using examples from three patients with unique clinical scenarios.

\section{Material and methods}

\section{Applicator design and production}

Computer aided design (CAD) software (Autodesk, Inc., San Francisco, CA, USA) was used to create a design file for a desired applicator shape, which is directly read by a 3D printer for production in thermoplastic. In our work-flow, the applicator shape is based on dimensions estimated from physical examination and imaging. Surface channels for interstitial needles are placed to allow optimal spacing between needles for high-dose-rate brachytherapy, approximately $1 \mathrm{~cm}$ apart. A central channel for a tandem, rod, or catheter can be incorporated as needed. We determined which plastics could be printed and sterilized, and we selected PC-ISO, a thermoplastic that is both USP Class VI approved and ISO-10993-1 rated (Stratasys Ltd., Eden Prairie, MN, USA). The material is also sterilizable and has high flexural and tensile strength properties that have made it a common choice for many medical applications, including ankle-foot orthoses, lumbar cages, and bone screw linking devices $[10,15,16,17,18]$. Documentation was obtained of an independent study describing the effectiveness of the STERRAD sterilization system (Ethicon, Inc., Sommerville, NJ, USA) for use with PC-ISO [19].

Multiple quality assurance and physical evaluations were performed on prototypes to ensure suitability for clinical use, which were reported in a separate technical paper [20].

\section{Patient 1}

Patient 1 was a 56 year-old woman with stage IA serous endometrial cancer who was referred for vaginal cuff brachytherapy following chemotherapy. Based on physical examination, the optimal applicator size was determined to be a $2.75 \mathrm{~cm}$ diameter vaginal cylinder. Because vaginal cylinder applicators at our institution are available only in $2.5,3$, or $3.5 \mathrm{~cm}$ diameter sizes, we decided to produce a custom-sized applicator using three-dimensional printing technology to better fit this patient's anatomy. This simple case also served as a proof of concept for the initial clinical implementation of a 3D printed custom vaginal applicator in our clinic. We used CAD software (Autodesk, Inc., San Francisco, CA, USA) to design a $2.75 \mathrm{~cm}$ diameter segmented cylinder with a single central channel. The cylinder was printed on a Fortus $400 \mathrm{mc}$ (Stratasys Ltd., Eden Prairie, MN, USA) three-dimensional printer, using PC-ISO biocompatible thermoplastic (Figure 1). The thermoplastic cylinder was sterilized using a STERRAD (Ethicon, Inc., Sommerville, NJ, USA) sterilization system.

At the time of treatment, two gold seed fiducial markers were inserted into the vaginal cuff prior to the first insertion. The sterilized cylinder was placed in the vagina, secured with a metal applicator clamp and baseplate, and the position was verified on scout film. Computed tomography images of the treatment area were obtained, and the applicator and organs at risk were contoured on Elekta Nucletron Oncentra Planning System (Verson 4.3; Elekta Instrument AB, Stockholm, Sweden). Three-dimensional inverse planning using inverse planning simulated annealing algorithm (IPSA) was used to develop a treatment plan to deliver 31.5 Gy over three fractions of 10.5 Gy per fraction, prescribed to the surface of the vagina. The applicator surface was used as a proxy for vaginal surface for the purposes of treatment planning. The IPSA class solution was designed with the goal of achieving 10.5 Gy to the surface of vagina. The length of vagina treated was based on physician's contour, which included the upper two-thirds of the vaginal canal. Coverage was verified by visual confirmation of the confluence of the 10.5 Gy isodose surface with the applicator surface. 3D volumetric doses were calculated for the bladder, rectum, bowel, and the target volume. The treatment was delivered using Elekta Nucletron microSelectron V3 remote afterloader (Elekta Instrument AB, Stockholm, Sweden).

\section{Patient 2}

Patient 2 was a 67 -year-old woman with vaginal cuff recurrence of stage IA grade 1 endometrioid endometrial cancer who had initially undergone total abdominal hysterectomy and bilateral salpingo-oophorectomy showing inner half myometrial invasion, grade 1 disease, and no lymphovascular invasion, who subsequently developed a vaginal cuff recurrence one year later and was treated with whole pelvis external beam radiotherapy to a dose of $45 \mathrm{~Gy}$, followed by vaginal cuff brachytherapy to a dose of $15 \mathrm{~Gy}$ over three fractions to the surface. Two years later, she developed a second vaginal cuff recurrence as well as distant metastatic disease and was referred for pallia-

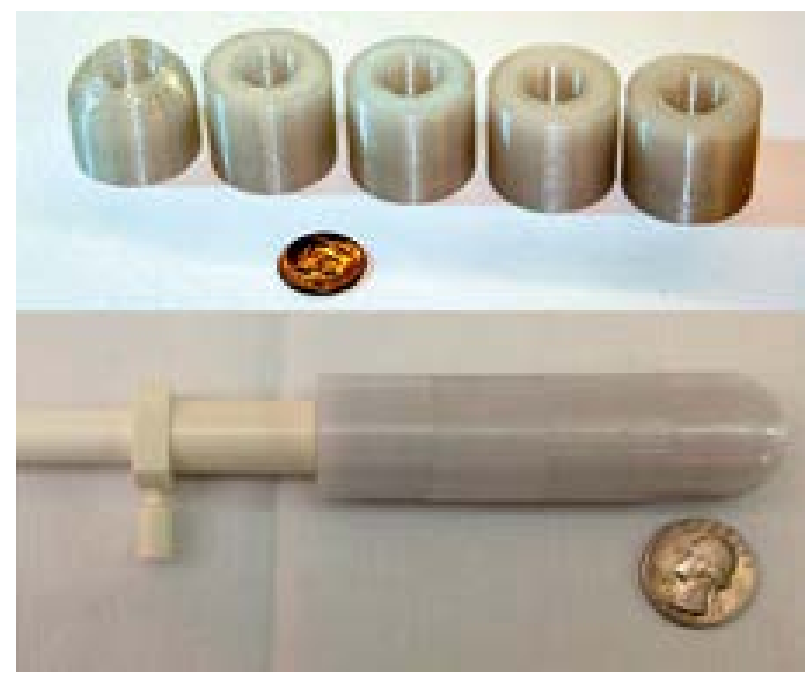

Fig. 1. Picture of the custom printed segmented cylinder sections with $2.75 \mathrm{~cm}$ diameter. The segments are stacked onto a plastic rod with a central channel for treatment as shown 
tive brachytherapy to a $2.4 \times 2.7 \mathrm{~cm}$ necrotic vaginal cuff mass causing progressive vaginal bleeding. Due to her prior treatment, the vaginal canal was very stenotic, and our narrowest commercial applicator could not be comfortably inserted. We therefore decided to print a vaginal cylinder applicator of $2 \mathrm{~cm}$ diameter, with a central catheter channel as well as six evenly spaced longitudinal surface grooves for interstitial brachytherapy to the vaginal cuff mass (Figure 2). Distance between surface grooves was $1 \mathrm{~cm}$. We used the same printing process and equipment as described for patient 1 .

At the time of treatment, two marker seeds were placed at the vaginal cuff. The patient underwent freehand trans-rectal ultrasound (TRUS)-guided interstitial brachytherapy. During this procedure, trans-rectal ultrasound was used to guide free-hand placement of flexible $30 \mathrm{~cm}$ interstitial catheters into the para-vaginal and vaginal cuff tissue. Additional catheters were placed along the surface and central channels of the vaginal cylinder to allow further dose modulation. The cylinder was secured to the vagina using sutures, and the catheters were secured to the cylinder using dental putty. For this patient, the final implant consisted of nine interstitial catheters, with six surface catheters around the cylinder, one central catheter, and two paravaginal catheters (Figure 3 ). She received 36 Gy over two implants, with 6 Gy per fraction and three fractions per implant prescribed to the CTV. CT-simulation and inverse planning were used, incorporating pre-operative MRI scan to guide tumor delineation. The CTV, bladder, rectum, and bowel were contoured. IPSA was used for planning. The IPSA class solution was designed to maximize the volume of the CTV receiving at least the prescription dose, minimize hotspots in the CTV $\left(\mathrm{V}_{150 \%}\right)$, and keep the $\mathrm{V}_{75 \%}$ of the bladder, rectum, and bowel below $1 \mathrm{~cm}^{3}$.

This patient also received two interstitial hyperthermia treatments, once during each implant. Hyperthermia was delivered using the BSD 500 System (BSD Medical Corporation, Salt Lake City, UT, USA), with MA-251 in-

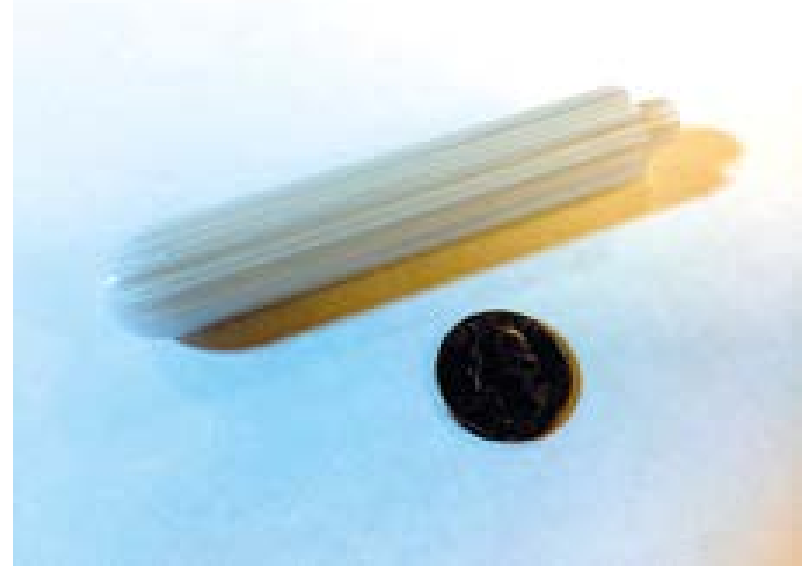

Fig. 2. Picture of the $2 \mathrm{~cm}$ diameter custom printed vaginal cylinder with six external catheter channels and one central catheter channel for use in interstitial brachytherapy and hyperthermia in a patient with a very narrow vaginal vault after prior surgery and radiotherapy terstitial microwave antenna and temperature monitoring sensors inserted within selected implant catheters. Target temperatures of 39.5-45 C for $60 \mathrm{~min}$ were prescribed, to be delivered immediately following treatment of either the first or second brachytherapy fraction. Hyperthermia treatment parameters included four or five microwave antennae with 4-6 W applied power per antenna, and four temperature sensors within adjacent catheters.

\section{Patient 3}

Patient 3 was a 37 year-old female with stage IIIA (cT2N2bM0) squamous cell carcinoma of the vulva, with a $6 \mathrm{~cm}$ fungating vulvar mass extending from the lower vagina to the anal verge, who underwent definitive chemoradiation. For treatment of the primary tumor, she received $45 \mathrm{~Gy}$ external beam radiation to the vulva with weekly cisplatin, followed by an interstitial brachytherapy boost to deliver an additional 18 Gy over three fractions in a single implant to the residual gross disease. She had a wide vaginal canal, and we printed a $3.5 \mathrm{~cm}$ diameter cylinder with a central channel for a tandem and ten surface grooves spaced $1.1 \mathrm{~cm}$ apart to accommodate peripheral surface catheters.

During the interstitial procedure, she underwent placement of gold seed markers to delineate tumor extent. She underwent subcutaneous insertion of four brachytherapy catheters, two through the left labia majora and two through the right labia majora. Nine additional flexible catheters were inserted transperineally into the bilateral paravaginal tissues (five on the left and four on the right) under palpation and ultrasound guidance. A uterine tandem and the custom printed $3.5 \mathrm{~cm}$ diameter vaginal cylinder were then placed, and ten additional catheters were inserted along the surface channels of the cylinder. The cylinder was tied in place with vaginal sutures, and the entire implant was secured together using dental putty around the cylinder and catheters. We followed the same applicator printing and treatment plan-

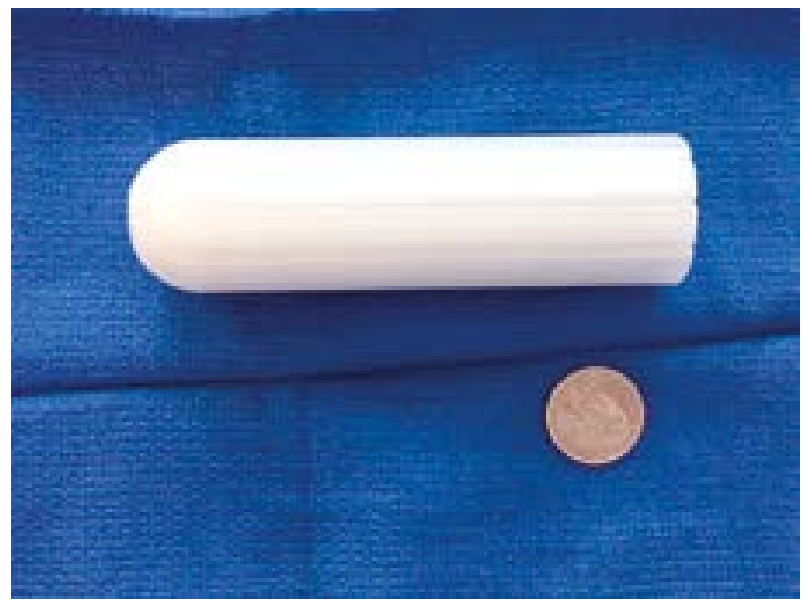

Fig. 3. Picture of the $3.5 \mathrm{~cm}$ diameter custom printed vaginal cylinder with ten external catheter channels and one central channel for a tandem for use in interstitial brachytherapy in a patient with a wide vaginal vault 
ning processes as described for patient 2, with additional planning parameters used to keep skin dose below 100\% of the prescription dose.

\section{Results}

\section{Patient 1}

The patient tolerated the $2.75 \mathrm{~cm}$ cylinder without discomfort, and there were no air-gaps seen at the time of CT-simulation. The plan is depicted in Figure 4. Dose volume histogram (DVH) parameters for each fraction were as follows: bladder $\mathrm{V}_{75 \%}=0.01 \mathrm{cc}, \mathrm{V}_{85 \%}=0 \mathrm{cc}, \mathrm{D}_{2 \mathrm{cc}}$ $=6.53 \mathrm{~Gy}$; rectum $\mathrm{V}_{75 \%}=0.95 \mathrm{cc}, \mathrm{V}_{85 \%}=0.06 \mathrm{cc}, \mathrm{D} 2 \mathrm{cc}=$ $7.31 \mathrm{~Gy}$; bowel $\mathrm{V}_{75 \%}=0 \mathrm{cc}, \mathrm{V}_{85 \%}=0 \mathrm{cc}, \mathrm{D} 2 \mathrm{cc}=4.68 \mathrm{~Gy}$; $\mathrm{CTV} \mathrm{V}_{100 \%}=90 \%$. The patient experienced no acute treatment effects other than mild fatigue for two weeks. After eighteen months of follow-up, she has had no evidence of disease on exam. She developed small adhesions at the vaginal apex six months after treatment, for which regular vaginal dilator use was encouraged. She had no other acute or late side effects of treatment.

\section{Patient 2}

The brachytherapy plan for implant 1 is depicted in Figure 5. Dose volume histogram parameters for each fraction were as follows: bladder $\mathrm{V}_{75 \%}=0.24 \mathrm{cc}, \mathrm{V}_{85 \%}=$ $0.01 \mathrm{cc}, \mathrm{D}_{2 \mathrm{cc}}=3.80 \mathrm{~Gy}$; rectum $\mathrm{V}_{75 \%}=0.24 \mathrm{cc}, \mathrm{V}_{85 \%}=0 \mathrm{cc}$, $\mathrm{D}_{2 \mathrm{cc}}=3.69 \mathrm{~Gy}$; bowel $\mathrm{V}_{75 \%}=0.4 \mathrm{cc}, \mathrm{V}_{85 \%} 0 \mathrm{cc}, \mathrm{D}_{2 \mathrm{cc}}=3.24 \mathrm{~Gy}$; CTV $\mathrm{V}_{100 \%}=91 \%$.

First hyperthermia treatment was delivered as prescribed using four interstitial applicators, with 4-6 W power applied to each antenna, generating target temperatures between $40-43.9^{\circ} \mathrm{C}$ for $60 \mathrm{~min}$ duration, and thermal dose between 1-49 $\mathrm{EM}_{43^{\circ} \mathrm{C}}$ (equivalent minutes at $43^{\circ} \mathrm{C}$ ). Second hyperthermia treatment utilized five interstitial applicators, with 4.5-5.5 $\mathrm{W}$ applied power, with temperatures of $39.4-44.8^{\circ} \mathrm{C}$, and thermal dose 2-28 $\mathrm{EM}_{43^{\circ} \mathrm{C}}$ achieved.

The patient had complete cessation of bleeding at her one-month follow-up appointment. She experienced fatigue and clear vaginal discharge for one month after treatment. MRI of the pelvis three months after treatment showed reduction in size of the vaginal mass, now measuring $1.9 \times 1.4 \mathrm{~cm}$. She died of progressive metastatic disease nine months after treatment without recurrence of vaginal bleeding.

\section{Patient 3}

The brachytherapy implant and treatment plan are depicted in Figure 6. Dose volume histogram parameters

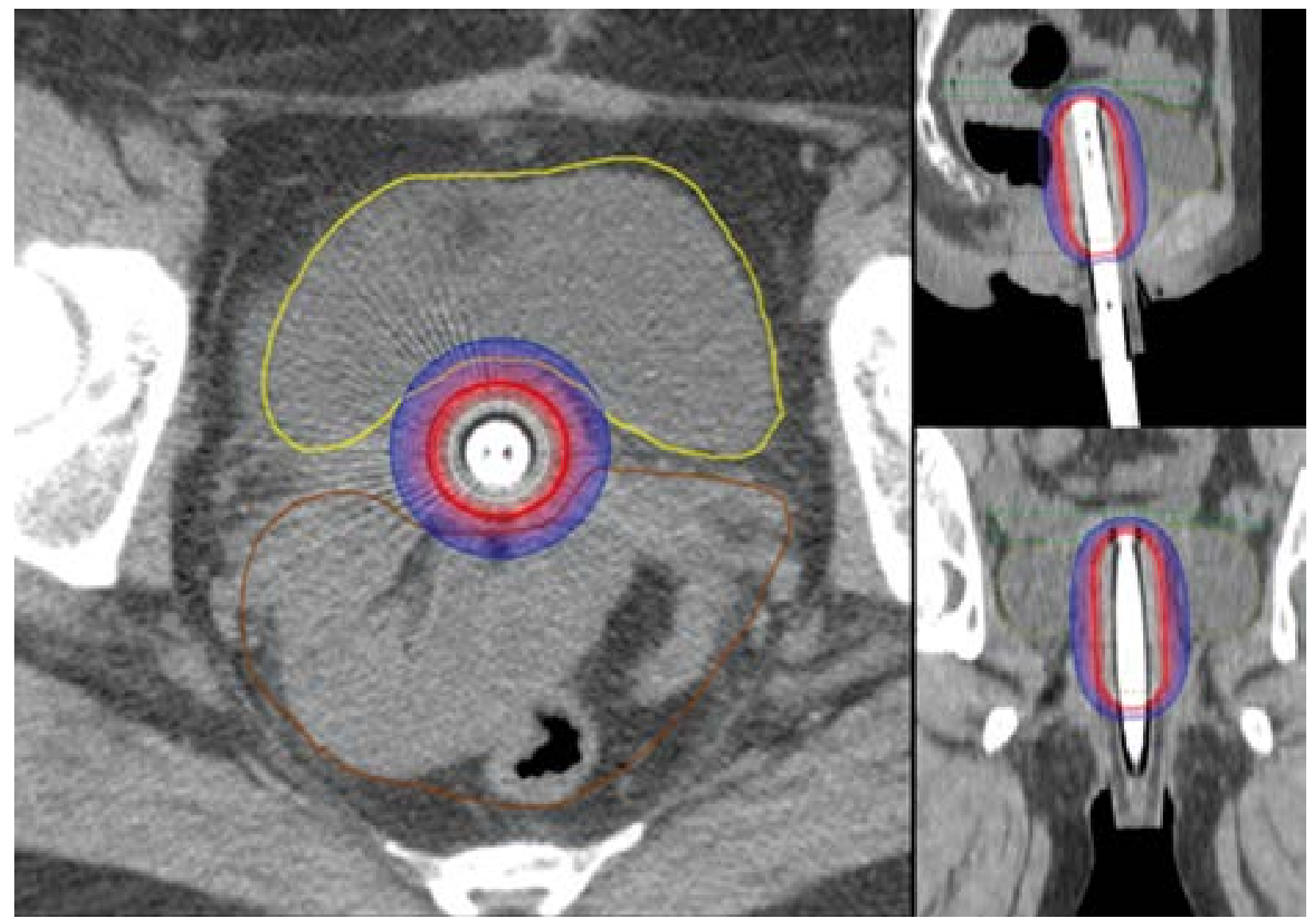

Fig. 4. Axial, sagittal, and coronal views of the brachytherapy treatment plan for patient 1 . Bold line indicates the gross target volume and is drawn at the surface of the cylinder, which is depicted with hyperintense central rod, around which are situated the segments of the cylinder. The $50 \%, 100 \%$, and $150 \%$ isodose lines, as well as organs at risk including the bowel, bladder, and rectum, are depicted 


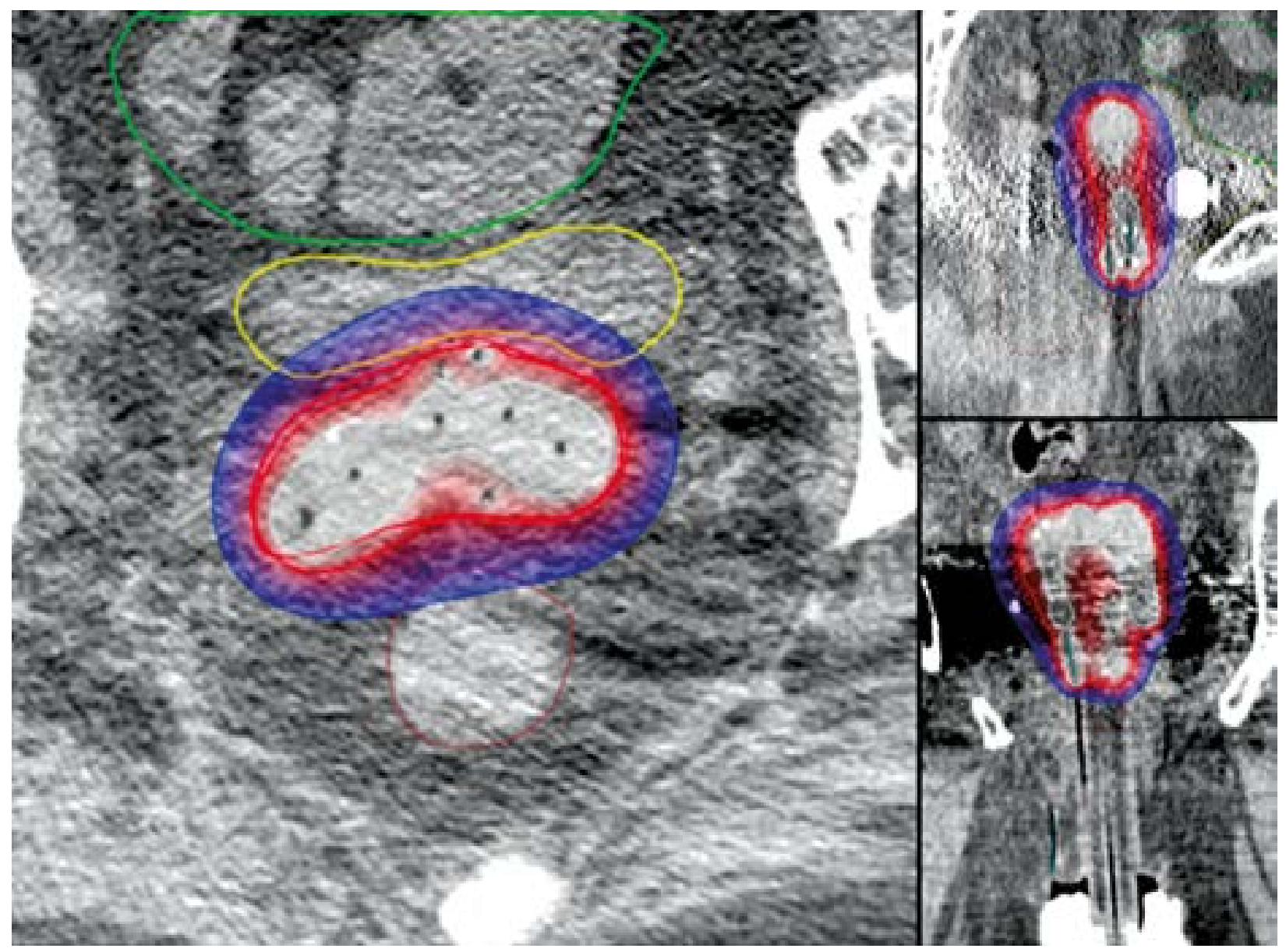

Fig. 5. Axial, sagittal, and coronal views of the brachytherapy treatment plan for patient 2 . Bold line indicates the gross target volume. The $50 \%, 100 \%$, and $150 \%$ isodose lines, as well as organs at risk including the bowel, bladder, and rectum, are depicted

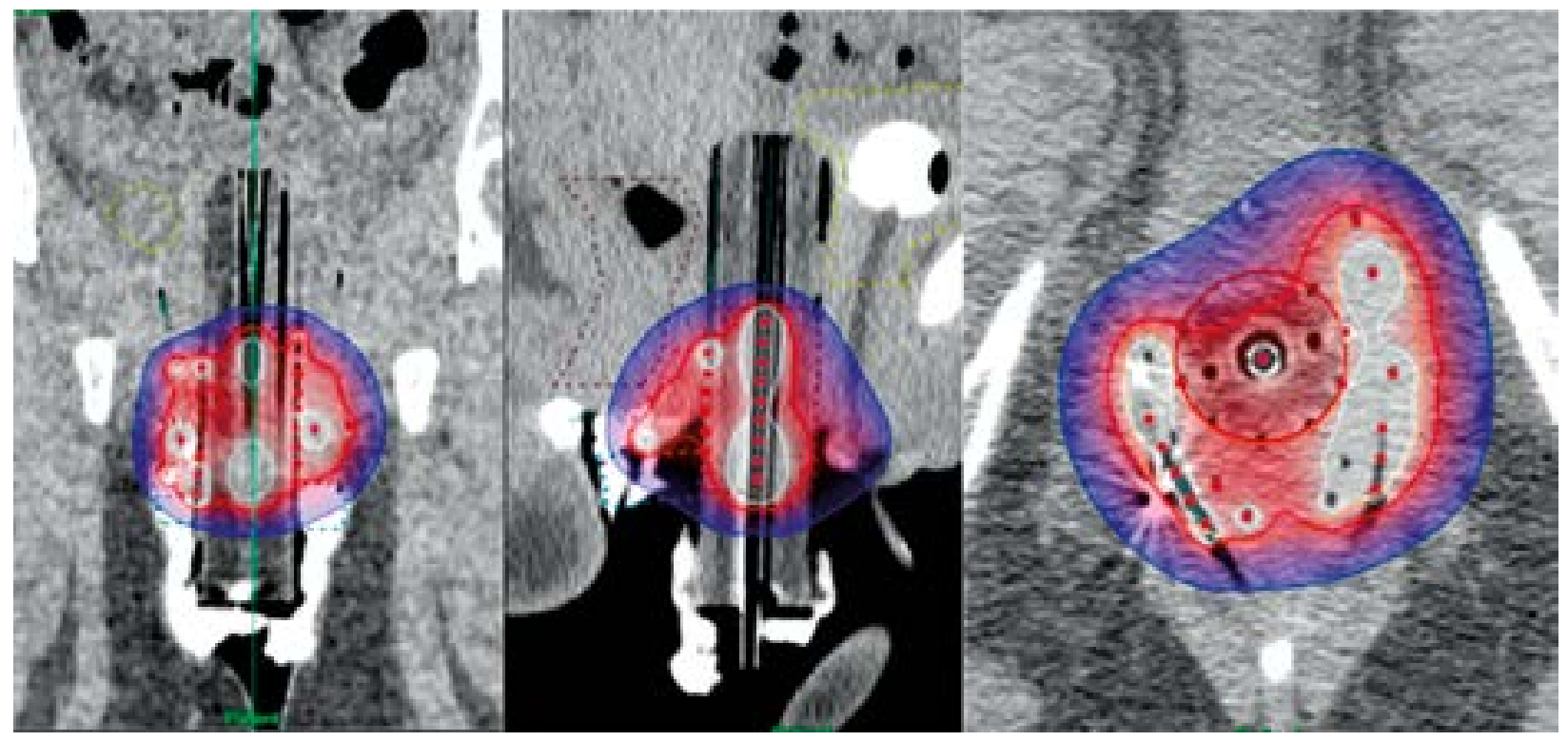

Fig. 6. Axial, sagittal, and coronal views of the brachytherapy treatment plan for patient 3 . Bold line indicates the gross target volume. The 50\%, 100\%, and 150\% isodose lines, as well as organs at risk including the bowel, bladder, and rectum, are depicted 
for each fraction were as follows: bladder $\mathrm{V}_{75 \%}=0 \mathrm{cc}$, $\mathrm{V}_{85 \%}=0 \mathrm{cc}, \mathrm{D}_{2 \mathrm{cc}}=1.32 \mathrm{~Gy}$; rectum $\mathrm{V}_{75 \%}=0.04 \mathrm{cc}, \mathrm{V}_{85 \%}=0 \mathrm{cc}$, $\mathrm{D}_{2 \mathrm{cc}}=3.02 \mathrm{~Gy} ;$ skin $\mathrm{D}_{0.1 \mathrm{cc}}=5.24 \mathrm{~Gy}, \mathrm{D}_{2 \mathrm{cc}}=3.08 \mathrm{~Gy}, \mathrm{D}_{1 \mathrm{cc}}=$ $3.63 \mathrm{cGy} ; \mathrm{CTV} \mathrm{V}_{100 \%}=95.4 \%$.

She is currently without evidence of disease after six months of follow-up. She developed acute grade 2 dysuria and pelvic and perineal pain requiring pyridium and narcotics for three weeks.

\section{Discussion}

We established the clinical feasibility of using 3D printed custom applicators for vaginal brachytherapy and, for two patients, in conjunction with interstitial brachytherapy. We have found these applicators useful for women whose anatomy falls outside the range of commercial applicators. In particular, patient 2 would not have been able to tolerate even the smallest commercial applicator at our institution $(2.5 \mathrm{~cm}$ diameter), and the printed applicator $(2.0 \mathrm{~cm}$ diameter $)$ made her implant feasible. Additionally, the wider diameter cylinder used for patient 3 allowed for more secure fit in a wide vaginal canal, while also permitting optimal placement of surface grooves for interstitial technique. In order to clinically implement these applicators while ensuring patient safety, multiple challenges in the design, manufacturing, and clinical application needed to be addressed.

With respect to applicator design, estimation of fit was based on physical exam. In the future, inverse applicator design based on imaging may be employed. Imaging techniques would need to incorporate either a real-time or post-processed expansion of the relaxed vaginal cavity. A volumetric contour could then be converted into a computer aided design file for 3D printing. One group recently reported on their process using CT scans with contrast-soaked vaginal gauze in place to delineate applicator shape for use with stereolithography [6].

The manufacturing process posed several challenges, including the need to perform proper QA of the custom applicators. When designing custom built applicators, the brachytherapy team takes on some of the roles traditionally filled by the vendor/manufacturer, therefore a fundamental understanding of the properties of the applicator is required. A suitable material needed to be selected and subjected to multiple quality assurance tests to document its feasibility for clinical use. Namely, the thermoplastic we selected needed to be biocompatible, sterilizable, CT-compatible, and have dose-attenuation properties similar to water in order to be compatible with our brachytherapy planning system.

We selected PC-ISO because at the time of this work it was one of a few materials with International Standard ISO-10993 Class VI Certification for biocompatibility, meaning it is FDA approved for temporary skin contact $[10,15]$, and it is sterilizable [19]. However, the current market of printing materials is expanding rapidly because of the wide range of medical applications for $3 \mathrm{D}$ printing and vendors are now providing a range of materials that are certified as both biocompatible and sterilizable. However, it is important for the clinic to verify that the sterilization systems available in your hospital has been validated by the vendor as a suitable system.

Computed tomography compatibility and dose-attenuation properties of the material are straightforward to determine; and once measured are valid for all uses of the material. The main issues to be careful with are the resolution of the printer used for manufacturing and the homogeneity of the final product. Though planning technology to handle tissue inhomogeneities are common in external beam planning, both Varian and Elekta brachytherapy treatment planning systems use TG-43 formalism, and assume all applicators and tissues are water. A straightforward method for determining the water equivalence of the chosen $3 \mathrm{D}$ printing material is to use gafchromic film embedded in a $3 \mathrm{D}$ printed sample of the material.

For readers interested in more detail, each of the QA elements discussed above are covered in our recently published technical paper [20].

During the planning process for patient 1 , we noticed that the density of PC-ISO is virtually tissue-equivalent on CT, making applicator delineation somewhat difficult during CT-based planning. This can be addressed by placing a small amount of contrast between the cylinder and condom cover prior to insertion at the time of simulation. Applicator delineation for patients 2 and 3 was facilitated by the surface grooves for peripheral catheters.

All three cases in this study were performed using CT for image-based planning. Magnetic resonance imaging, however, is beginning to see more use in gynecological brachytherapy because of its enhanced soft tissue contrast MRI, increased availability, and publication of multiple sets of guidelines regarding its use in gynecologic brachytherapy over the past decade. It should be noted that while CT compatibility and photon attenuation are intimately related, the same is not true for MRI. Materials should be independently verified to not have any ferromagnetic properties. In addition, if the custom applicator design is to have internal channels for the brachytherapy catheters, it is important that the MR imaging can resolve them for digitization. If this is not the case, a method for introducing contrast into the brachytherapy channels should be developed.

\section{Conclusions}

We used 3D printing technology to produce biocompatible, sterilizable, custom applicators for patients undergoing gynecologic brachytherapy for whom standard applicators would have resulted in suboptimal implants. 3D printing enabled rapid production of applicators with excellent fit and optimized interstitial needle placement to enable target coverage, normal tissue sparing, and implant stability. In the future, this clinical workflow could easily be adapted to allow more complicated surface contours and irregular shaped applicators inversely designed from imaging. 


\section{Disclosure}

Authors report no conflict of interest.

\section{References}

1. Richardson S, Palaniswaamy G, Grigsby PW. Dosimetric effects of air pockets around high-dose rate brachytherapy vaginal cylinders. Int J Radiat Oncol Biol Phys 2010; 78: 276-279.

2. Cameron AL, Cornes P, Al-Booz H. Brachytherapy in endometrial cancer: quantification of air gaps around a vaginal cylinder. Brachytherapy 2008; 7: 355-358.

3. Pinnaduwage DS, Cunha JA, Weinberg V. A dosimetric evaluation of using a single treatment plan for multiple treatment fractions within a given applicator insertion in gynecologic brachytherapy. Brachytherapy 2013; 12: 487-494.

4. Magné N, Chargari C, SanFilippo N et al. Technical aspects and perspectives of the vaginal mold applicator for brachytherapy of gynecologic malignancies. Brachytherapy 2010; 9: 274-277.

5. Miller DA, Richardson S, Grigsby PW. A new method of anatomically conformal vaginal cuff HDR brachytherapy. Gynecol Oncol 2010; 116: 413-418.

6. Wiebe E, Easton $H$, Thomas $\mathrm{G}$ et al. Customized vaginal vault brachytherapy with computed tomography imaging-derived applicator prototyping. Brachytherapy 2015; 14: 380-384.

7. Chow LK, Cheung LK. The usefulness of stereomodels in maxillofacial surgical management. J Oral Maxillofac Surg 2007; 65: 2260-2268.

8. Anchieta M, Quaresma M, de Salles F. Rapid Prototyping Applied to Maxillofacial Surgery. In: Advanced Applications of Rapid Prototyping Technology in Modern Engineering. Hoque ME, editor. InTech, Rijeka 2011.

9. Cohen A, Laviv A, Berman P et al. Mandibular reconstruction using stereolithographic 3-dimensional printing modeling technology. Oral Surg Oral Med Oral Pathol Oral Radiol Endod 2009; 108: 661-666.

10. Schrank ES, Hitch L, Wallace K et al. Assessment of a virtual functional prototyping process for the rapid manufacture of passive-dynamic ankle-foot orthoses. J Biomech Eng 2013; 135: 101011-101017.

11. Winder J, Bibb R. Medical rapid prototyping technologies: state of the art and current limitations for application in oral and maxillofacial surgery. J Oral Maxillofac Surg 2005; 63: 1006-1015.

12. Winder J, Cooke RS, Gray J et al. Medical rapid prototyping and 3D CT in the manufacture of custom made cranial titanium plates. J Med Eng Technol 1999; 23: 26-28.

13. Choi JY, Choi JH, Kim NK et al. Analysis of errors in medical rapid prototyping models. Int J Oral Maxillofac Surg 2002; 31: 23-32.

14. Webb PA. A review of rapid prototyping (RP) techniques in the medical and biomedical sector. J Med Eng Technol 2000; 24: 149-153.

15. Novakova-Marcincinova L, Novak-Marcincin J. Experimental testing of materials used in fused deposition modeling rapid prototyping technology. Advanced Materials Research 2013; 740: 597-602.

16. Koo S, Hargreaves BA, Gold GE et al. Fabrication of custom-shaped grafts for cartilage regeneration. Int J Artif Organs 2010; 33: 731-737.

17. Aherwar A, Singh A, Patnaik A. A review paper on rapid prototyping and rapid tooling techniques for fabrication of prosthetic socket. High Value Manufacturing. Adv Res Virt Rapid Prot 2014; pp. 345-353.

18. Groscurth RC, Groscurth, SU, inventors, Bone screw linking device. United States patent application number US 12/882,800. September 15, 2010.
19. Perez M, Block M, Espalin D et al. Sterilization of FDM-Manufactured Parts. 23 ${ }^{\text {rd }}$ Annual International Solid Freeform Fabrication Symposium - An Additive Manufacturing Conference; August 6-8, 2012; Austin, Texas. pp. 285-296.

20. Cunha JA, Mellis K, Sethi R et al. Evaluation of PC-ISO for Customized, 3D Printed, Gynecologic 192-IR HDR Brachytherapy Applicators. J Appl Clin Med Phys 2015; 16: 5168. 